\title{
Study of heat transfer processes in the flowing part of hypersonic air-ramjet engine
}

\author{
Eugene Maslov ${ }^{1,2 *}$, Irina Zharova ${ }^{1}$, Valery Faraponov ${ }^{1}$, Eugene Kozlov ${ }^{1}$, and Vladislav \\ Matskevich $^{1}$ \\ ${ }^{1}$ National Research Tomsk State University, 634050 Tomsk, Russia \\ ${ }^{2}$ National Research Tomsk Polytechnic University, 634050 Tomsk, Russia
}

\begin{abstract}
The technique and results of the experimental-theoretical study of gas dynamics, heat transfer and the structure of gas flow in the flowing channel of a model hypersonic air-ramjet engine are presented for Mach numbers $\mathrm{M}=(5 ; 6)$.
\end{abstract}

\section{Introduction}

At hypersonic flight speeds of rockets at low altitudes, it is supposed to use solid fuel airramjet engine. Wherein, the air from the atmosphere, which enters through the air intake into the flowing channel of the engine with velocities $M>5$ [1], is used as an oxidizer in the organization of combustion. The processes of combustion and burning-out (possibly, uneven) of the surface of the solid fuel element are determined by gas-dynamic and thermal parameters of flow in the flowing part of the engine. Thus, for design of engine facility, it is necessary to have of experimental studies data from that allow one to predict these parameters with a high degree of veracity for concrete designs [2]. It is especially important to solve the problem of assessing the thermal state of the flowing channel in the initial period after the switching-on of an installation. The presence of predictive estimates of the thermal state of the tract at the design stage of a specific design is necessary for a wellfounded choice of the ignition method and the provision sustainable combustion of solid fuel. It is advisable to conduct preliminary studies with laboratory models, since full-scale stand experiments are costly.

The purpose of the study was to obtain objective information about the thermal state of the walls of the flowing channel of a model hypersonic air-ramjet engine at Mach numbers $\mathrm{M}=(5,6)$.

\section{Technique of the experimental study}

An experimental study was carried out on a pulsed aerodynamic installation with heating of gas (up to $T_{e} \approx 700 \mathrm{~K}$ ) [3-5]. The aerodynamic installation provides the speed of the inflowing stream in the range of Mach numbers $M=(2 \div 7)$. Steel axisymmetric profiled

\footnotetext{
* Corresponding author: maslov_eugene@mail.ru
} 
nozzles [4] were used to create a hypersonic flow for Mach numbers $M=(5,6)$. The experiments were conducted on an axisymmetric model of an air-ramjet engine (Fig. 1, a).

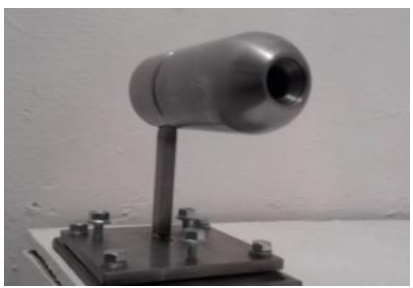

(a)

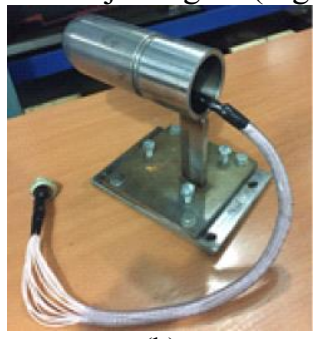

(b)

Fig. 1. General view of model (a), and model with the installed thermo-probe (b).

The measurement of the gas temperature in the flowing channel was carried out by a thermocouple method [6]. A thermo-probe was manufactured for measure of the temperature along the wall of the model flowing channel. The thermos-probe represents a set of fluoroplastic rings of the equal diameter. Eight copper rings with a chromel-copel thermocouple were attached to the inner surface of each ring. The view of the axisymmetric model with the installed thermo-probe is shown in Fig. 1, b. The scheme (Fig. 2, a) and photographs of the thermo-probe in assembled form (Fig. 2, b) and the temperature sensor (Fig. 2, c) are shown.

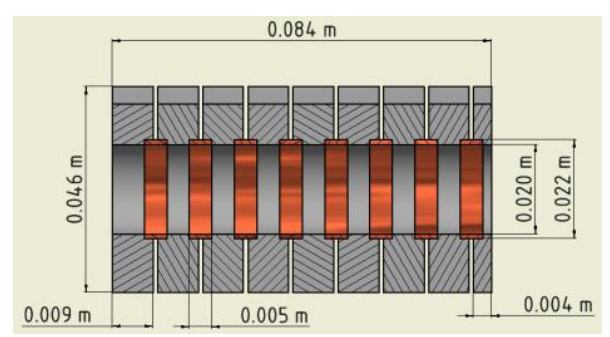

(a)

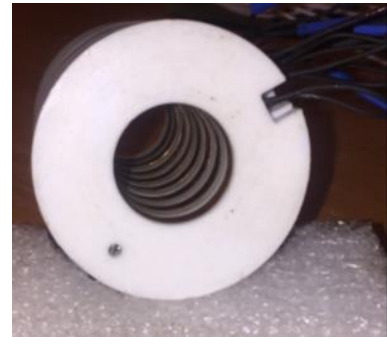

(b)

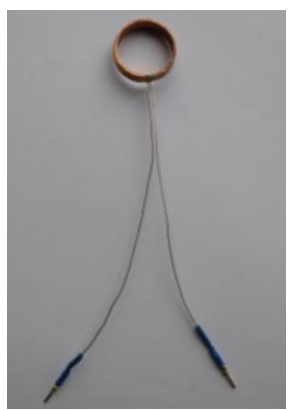

(c)

Fig. 2. Scheme of the thermo-probe (a), the thermo-probe fully assembled (b) and the temperature sensor (c).

The temperature in the prechamber and in the exit section of the model was measured with the help of special temperature sensors during process of the experiments. The view of placed in the working part of the aerodynamic installation the axisymmetric model in complex with temperature sensors is shown in Fig. 3. 


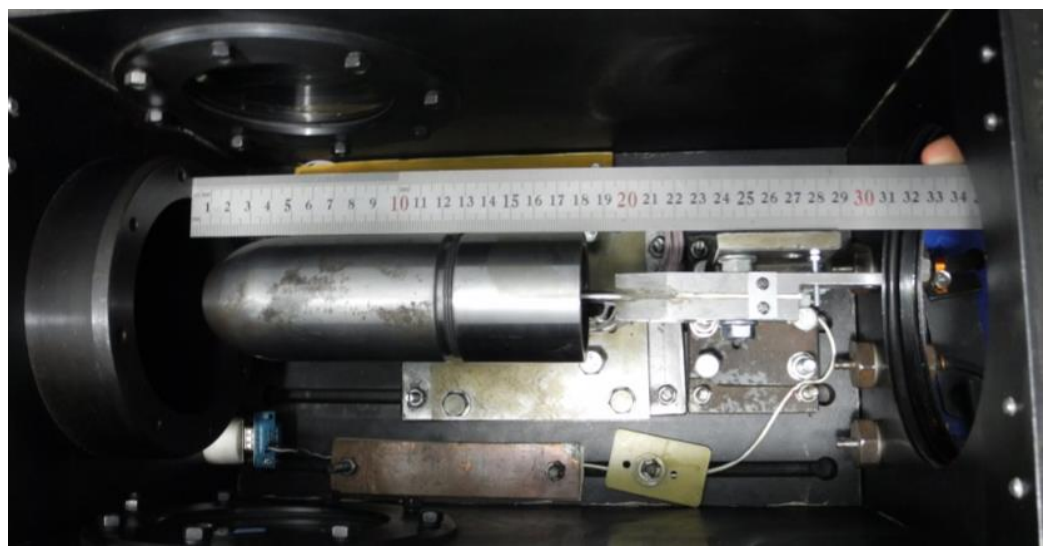

Fig. 3. Axisymmetric model located in the working part of the aerodynamic installation in complex with temperature sensors.

\section{Results of the experimental study}

Series of thermogasdynamic experiences were carried out for each Mach number $M=(5,6)$. The measured values of the static temperature in the prechamber and the stagnation temperature in the exit section of the model are shown in Fig. $4,5$.

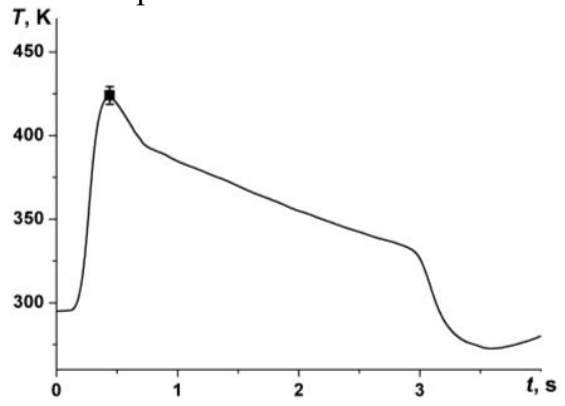

(a)

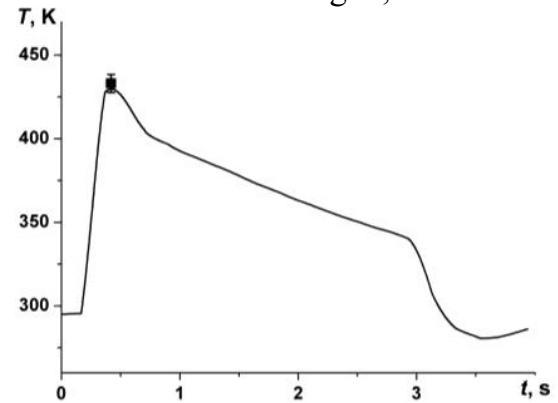

(b)

Fig. 4. Dependence of the static temperature in the prechamber on time at $M=5$ (a) and $M=6$ (b).

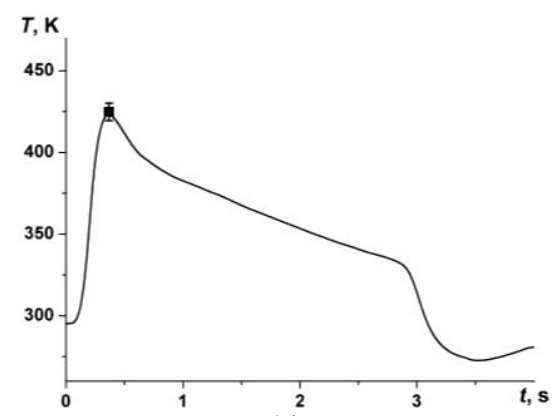

(a)



(b)

Fig. 5. Dependence of the stagnation temperature in the exit section of the axisymmetric model on time for $M=5$ (a) and $M=6$ (b).

The temperature measurement data for flowing around an axisymmetric model are given in Fig. 6, a, 7, a, $M=(5,6)$. The data were obtained in one of the tests in a series of ten experiments. These are conditions for conducting a series of experiments: the heater 
temperature is $675 \mathrm{~K}$, the work duration of the aerodynamic installation is $3 \mathrm{~s}$. The maximum temperature values averaged over a series of experiments at the corresponding Mach numbers are given in Fig. 6, b, 7, b ( $x$ is the distance from the initial section of the model to the hot junction along the thermo-probe).

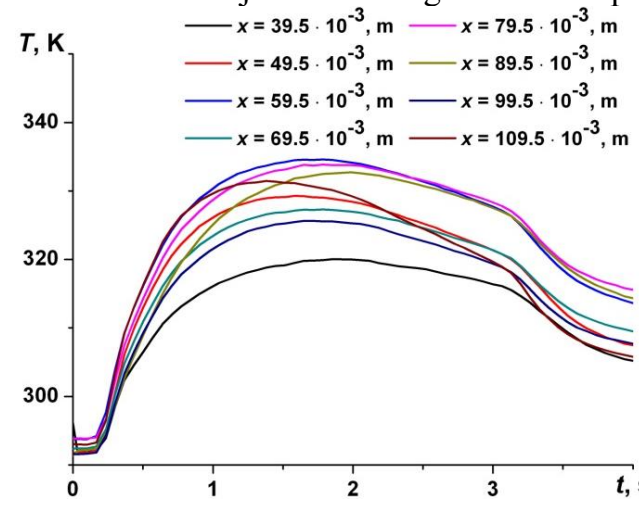

(a)

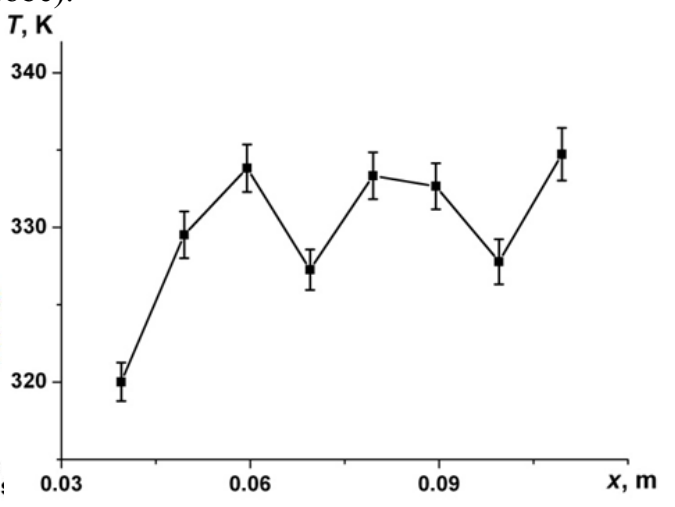

(b)

Fig. 6. Typical temperature distribution along the surface of the flowing channel (a) and the maximum temperature values averaged over a series of experiments (b), $M=5$.

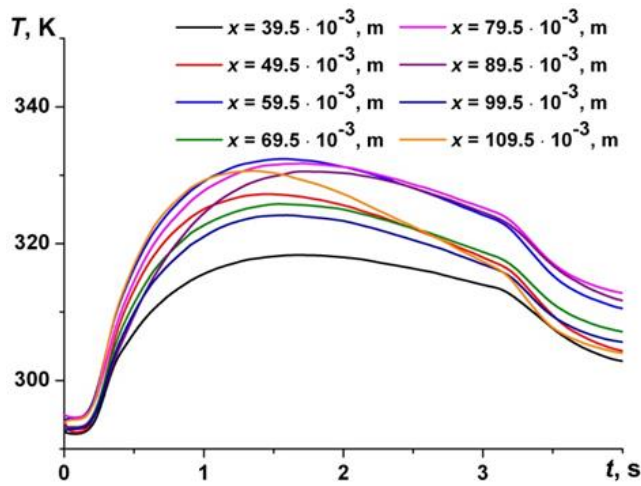

(a)

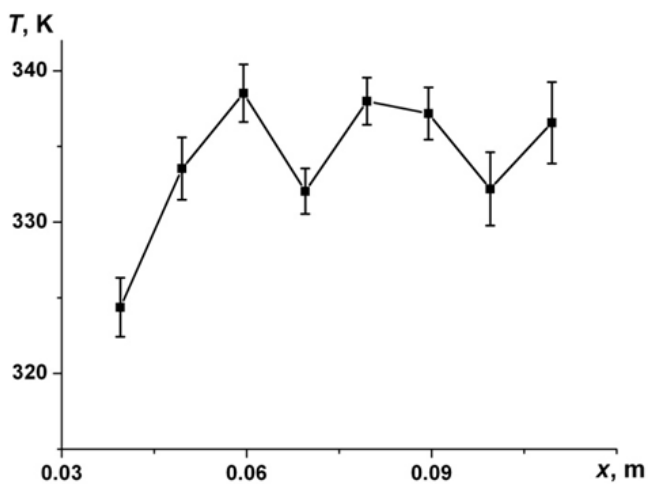

(b)

Fig. 7. Typical temperature distribution along the surface of the flowing channel (a) and the maximum temperature values averaged over a series of experiments (b), $M=6$.

\section{Discussion of the results}

Veracity of the data obtained with the help of the developed technique of experimental research is confirmed by their comparison with the results of calculations. Numerical analysis for $\mathrm{M}=(5 ; 6)$ was carried out on the basis of a mathematical model [7]. Numerically obtained distributions of Mach number and the temperature along the axis of symmetry of the flowing channel of the model for $\mathrm{M}=5$ are shown in Fig. 8. When $x=0.042 \mathrm{~m}$ ( $x$ is the distance from the initial model section to fixed point), the value of the Mach number was obtained experimentally (the point in Fig. 8, a) [5]. Comparison of the numerical and experimentally obtained Mach number values showed that the relative error is less than $10 \%$. The nature of the temperature change along the axis of the flowing channel (Fig. 8, b) correlates with the temperature measurement data along the thermoprobe surface and adequately describes the physical process [4]. 


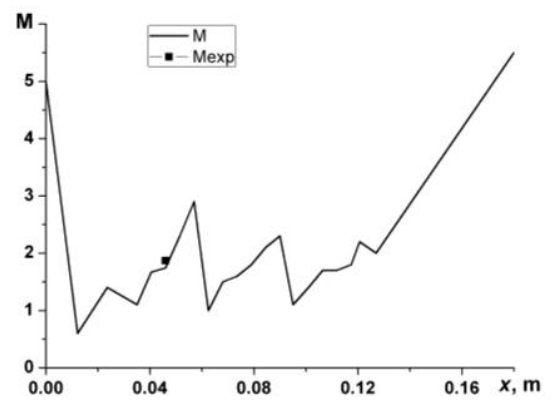

(a)

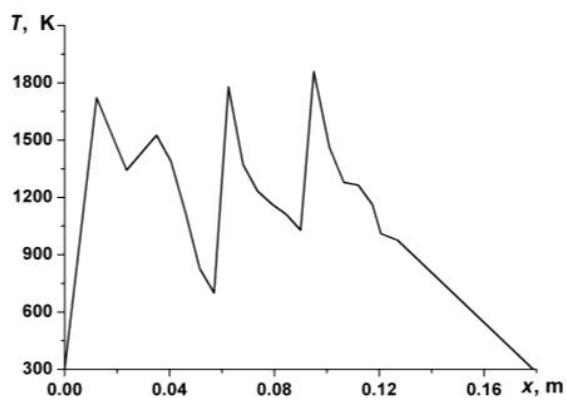

(b)

Fig. 8. Distributions of the Mach number (a) and the temperature (b) along the axis of symmetry of the flowing channel of the axisymmetric model, $\mathrm{M}=5$.

The correlation of oscillatory regimes by Mach number, and temperature on the symmetry axis is noted. The Mach number decreases in the zones of compression shock, and the temperature increases. The Mach number increases in the decompression zones, and the temperature decreases.

\section{Conclusion}

The thermo-probe is developed, designed and implemented. The thermo-probe is equipped by temperature sensors with chromel-copel thermocouples. The working range of the measuring temperatures is $T=(200 \div 600) \mathrm{K}$.

The experimentally-calculating technique of studying gas dynamics, heat transfer and the structure of gas flow in the flowing channel of hypersonic air-ramjet engine are proposed.

Objective data about the heat transfer characteristics in the flowing part of the axisymmetric model of the air-ramjet engine were obtained for Mach numbers $M=(5,6)$ on the basis of the developed technique.

This research was supported by grant (No. 8.2.09.2018) from "The Tomsk State University competitiveness improvement program".

\section{References}

1. L. S. Yanovski, Integral ramjet solid propellant air-jet engines. Fundamentals of theory and calculation: Reference book (Moscow, Akademkniga, 2006) [in Russian]

2. B. V. Orlov, G. Yu. Mazing, A. L. Reidel, Basics of design of rocket-ramjet engines for unmanned aerial vehicles (Moscow, Mashinostroenie, 1967) [in Russian]

3. V. I. Zvegintsev, Gasdynamic installations of short-term action: Reference book. (Novosibirsk, Parallel, 2014) [in Russian]

4. E. Maslov, I. Zharova, V. Klochikhin, MATEC Web Conf. 23 (2015)

5. E. Maslov, I. Zharova, Ye. Kozlov, V. Faraponov, N. Savkina, N. Zolotorev, V. Matskevich, MATEC Web Conf. 115 (2017)

6. E. A. Maslov, I. K. Zharova, A. S. Zhukov, Materials of the All-Russian Conference "XXXII Siberian Thermophysical Seminar": Reference book (Institute of Thermophysics SB RAS, Novosibirsk, 2015) [in Russian]

7. V. A. Arkhipov, A. G. Egorov, S. V. Ivanin, E. A. Maslov, O. V. Matvienko, Combust. Explos. Shock Waves 46, 1 (2010) 